\title{
Low lymphatic vessel density associates with chronic rhinosinusitis with nasal polyps*
}

\author{
A.Luukkainen'1, M. Seppälä', J. Renkonen', R. Renkonen 1,2, J. Hagström¹,2, \\ H.Huhtala ${ }^{3}$, M. Rautiainen ${ }^{4}$, J. Myller ${ }^{5}$, T. Paavonen ${ }^{6,7}$, A. Ranta ${ }^{8}$, T. Torkkeli9, \\ Rhinology 55: 181-191, 2017 \\ S.Toppila-Salmi ${ }^{1,10}$ \\ https://doi.org/10.4193/Rhino16.007 \\ ${ }^{*}$ Received for publication: \\ ' Haartman institute, University of Helsinki, Helsinki, Finland \\ January 20, 2016 \\ 2 HUSLAB, Helsinki University Hospital, Helsinki, Finland \\ Accepted: March 31, 2017 \\ ${ }^{3}$ Faculty of Health Sciences, University of Tampere, Tampere, Finland \\ ${ }^{4}$ Department of Otorhinolaryngology, Tampere University Hospital. Tampere, Finland \\ ${ }^{5}$ Department of Otorhinolaryngology, Päijät-Häme Central Hospital, Lahti, Finland \\ ${ }^{6}$ Department of Pathology, University of Tampere, Tampere, Finland \\ Fimlab Laboratories Ltd, Tampere, Finland \\ ${ }^{8}$ Department of Surgery, Tampere City Hospital, Tampere, Finland \\ ${ }^{9}$ Department of Otorhinolaryngology, Mikkeli Central Hospital, Mikkeli, Finland \\ ${ }^{10}$ Skin and Allergy Hospital, University of Helsinki and Helsinki University Hospital, Helsinki, Finland
}

\begin{abstract}
Objectives: Chronic rhinosinusitis with and without nasal polyps (CRSwNP and CRSsNP) and antrochoanal polyps (ACP) are different upper airway inflammation phenotypes with different pathomechanisms. In order to understand the development of tissue edema, the present study aimed to evaluate lymphatic vessel density in CRSsNP, CRSwNP and ACP.
\end{abstract}

Materials and Methods: 120 retrospective nasal and maxillary sinus specimens were stained immunohistochemically with a von Willebrand factor polyclonal antibody recognizing vascular and lymphatic endothelium, and with a podoplanin monoclonal antibody recognizing lymphatic endothelium. Vessels were studied by microscopy in a blinded fashion, and the vessel density and the relative density of lymphatic vessels were calculated. Patient characteristic factors and follow-up data of in average 9 years were collected from patient records.

Results and Conclusion: In the nasal cavity, the low absolute and relative density of vessels and of lymphatic vessels was associated with CRSwNP and ACP tissues compared to control inferior turbinate. This was observed also in the inflammatory hotspot area. In the maxillary sinus, lower absolute and relative density of lymphatic vessels associated with the CRSwNP phenotype. High lymphatic vessel density in polyp tissue associated with the need for revision CRS-surgery. As a conclusion, low density of lymphatic vessels distinguished patients with CRSwNP not only in the hotspot area of polyp tissue, but also in maxillary sinus mucosa. Yet, higher lymphatic vessel density seems to associate with polyp recurrence. Further studies are still needed to explore if formation of nasal polyps could be diminished by intranasal therapeutics affecting lymphangiogenesis.

Key words: sinusitis, nasal polyps, lymphangiogenesis, reoperation, immunohistochemistry

\section{Introduction}

Chronic rhinosinusitis (CRS) is a heterogeneous group of inflam- matory diseases of the nose and paranasal sinuses lasting for at least 12 weeks ${ }^{(1)}$. It is a significant health problem, with a

Abbreviations: ACP: Antrochoanal polyp; AERD: Aspirin-exacerbated respiratory disease; CD: Cluster of Differentiation; CRS: Chronic rhinosinusitis; CRSsNP: CRS without nasal polyps; CRSwNP: CRS with nasal polyps; ESS: endoscopic sinus surgery; mAb: monoclonal antibody; NP: nasal polyp; Th1: T-helper cell 1; VEGF: vascular endothelial growth factor; vWf: von Willebrand factor; RDLV: relative density of lymphatic vessels 
prevalence of around 7-11\% ${ }^{(1-3)}$. It is frequently associated with asthma but their inter-relationship is poorly understood ${ }^{(1,4)}$. CRS with nasal polyps (CRSwNP) and without (CRSsNP) are considered to be phenotypes of CRS with possibly different aetiologies and pathomechanisms. However, they may also be interpreted as different degrees of inflammation ${ }^{(1)}$. Nasal polyps (NP) appear as oedematous masses originating from the middle meatus and affect between 1 and $4 \%$ of the general population ${ }^{(1)}$.

Sinus specimens obtained from patients suffering from CRSsNP are generally characterised by basement membrane thickening, goblet cell hyperplasia, subepithelial oedema, abundant mononuclear cells and few eosinophils ${ }^{(1,5)}$. Histomorphological characterisation of CRSWNP reveals epithelial damage, goblet cell metaplasia, a thickened basement membrane and oedematous to sometimes fibro-inflammatory tissue ${ }^{(6,7)}$. CRSsNP is characterized by a T-helper cell 1 (Th1) polarization with high levels of interferon-gamma and transforming growth factor beta. Caucasian CRSwNP is characterised by a Th2-skewed eosinophilic inflammation with high interleukin 5 levels ${ }^{(8)}$, and reduced transforming growth factor beta, whilst over $50 \%$ of East Asian polyps are characterized with a Th1/Th17, neutrophilic dominant pattern ${ }^{(9-12)}$.

Antrochoanal polyps (ACP) are benign polypoid lesions arising from the maxillary antrum and which extend into the choana. They occur more commonly in children and young adults and are almost always unilateral ${ }^{(13)}$. Human papillomavirus type 16 has been detected at higher frequencies in ACP ${ }^{(14)}$. Quantification of blood and lymphatic vessel density has previously been used to understand cancer biology and used as a prognostic factor in melanoma and head and neck cancer, for example ${ }^{(15,16)}$. Increased angiogenesis and density of blood vessels as documented by positive $\mathrm{CD} 34$ and pro-angiogenic factor vascular endothelial growth factor (VEGF) has been reported respectively in adult CRSWNP and ACP patients ${ }^{(17)}$ and in CRSwNP in children ${ }^{(18)}$. The sub-epithelial submucosa of the lungs of asthmatic patients has been documented to have an increased vascular density, both ex vivo and in vivo and airway tissue to contain increased levels of VEGF $(19,20)$.

There are several antibodies which recognize blood and/or lymphatic vessel antigens. von Willebrand factor (vWf) is a large multimeric glycoprotein, with a mass ranging from $500 \mathrm{kDa}$ up to over $10000 \mathrm{kDa}{ }^{(21)}$. The encoding gene of $\mathrm{vWf}$ is located in chromosome 12p13.2. vWf is produced by endothelial cells and megakaryocytes ${ }^{(22)}$. The primary product of the gene is a 2813 amino acids protein; which is composed of a signal peptide of 22 amino acids, a propeptide of 741 amino acids and a mature vWF molecule containing 2050 amino acids (21). vWf is expressed by blood endothelium and also weakly expressed on lymphatic endothelium ${ }^{(23-27)}$.

Monoclonal antibody podoplanin (clone D2-40) recognizes podoplanin in lymphatic endothelium. D2-40 was originally recognized as an oncofetal antigen, M2A antigen, which is an Olinked sialoglycoprotein with a simple mucin-type carbohydrate epitope associated with germ cell neoplasms ${ }^{(28,29)}$. Currently, it is known that podoplanin and M2A antigen are identical proteins (29). Podoplanin is expressed in lymphatic endothelial cells but is not expressed in blood endothelial cells ${ }^{(30)}$. Therefore it is a specific marker for lymphatic endothelium ${ }^{(30)}$. In order to better understand these pathways, this study aimed to evaluate lymphatic vessel density in CRS subtypes and ACP.

\section{Materials and methods}

Clinical samples

This study was performed at the Departments of Otorhinolaryngology and Pathology, Tampere University Hospital, at the Department of Otorhinolaryngology, Mikkeli Central Hospital, and at Haartman institute, University of Helsinki, Finland. The study protocol was approved by the Ethical Committee of the Pirkanmaa Hospital District. The Ethical Committee did not require informed consent for this follow-up study. The formalin-fixed paraffin-embedded polyp samples were collected retrospectively from the archives of the hospital from 37 patients who had been treated for CRS and 9 for ACP 1999 - 2007 as previously described ${ }^{(31)}$. Control inferior turbinate samples were collected prospectively from 19 healthy non-smoking volunteers in 200507 as previously described ${ }^{(31)}$. The maxillary sinus samples form 44 CRS and 11 bimaxillary osteotomy patients (controls) were collected prospectively in 2001-04 as previously described ${ }^{31,32)}$. Maxillary sinus specimens were taken from the latero-inferior wall of the maxillary sinus. All the subjects were Caucasian. Subject groups are shown in Tables 1-2. The inclusion criteria of patients were: diagnosis of CRSsNP, CRSwNP, or ACP based on European Position Paper on rhinosinusitis and nasal polyps 2012 criteria of symptoms, endoscopic and sinus computed tomography findings ${ }^{(1)}$. The exclusion criteria were cystic fibrosis and diseases with a severe impact on general immunity. The exclusion criteria of control subjects were: sinonasal disease (except mild allergic rhinitis) or any other disease requiring constant medication. Diagnosis of allergic rhinitis was based on skin prick test positivity and typical symptoms. Diagnosis of asthma was based on clinical features and pulmonary function tests. Diagnosis of Aspirin-exacerbated respiratory disease (AERD) was made on the basis of a history of wheezing or asthma attacks precipitated by non-steroidal anti-inflammatory drugs. All samples were reviewed by a pathologist. The characteristics, collected from patient records, are shown in Tables 1-2.

The follow-up data of sinonasal operations and time of surgery was collected form patient records of Tampere University Hospital, Tampere City Hospital, and Mikkeli Central Hospital in 2014-15 in average 9 years after the time of sampling. None of 
the subjects had used Aspirin desensitization, allergen immunotherapy, or anti IgE therapy prior to or during the sampling or during the follow-up.

\section{Tissue handling and immunohistochemistry} von Willebrand factor and podoplanin staining were performed in 120 samples. The samples were cut into $4-5 \mu \mathrm{m}$ thick paraffin sections and were placed on Superfrost Plus microscope slides (Menzel-Gläser, Braunschweig, Germany). Fully automated immunostaining was performed by Ventana BenchMark LT Automated IHC Stainer (Ventana Medical System, Tucson, Arizona, USA). Ultraview Universal DAB detection kit (catalog No. 760-500, Ventana Medical System) was used. Ventana EZ Prep solution (catalog No 950-100, Ventana Medical System) was used for deparaffinisation. For epitope retrieval CC1:Tris -EDTA buffer pH 8.0 (catalog No 950-124, Ventana Medical System) was used at $95^{\circ} \mathrm{C}$ to $100^{\circ} \mathrm{C}$ for 30 minutes with paraffin embedded tissue sections. Endogenous peroxidase was blocked with UV-Inhibitor 3\% H202 (Ventana Medical System) for 4 minutes at $37^{\circ} \mathrm{C}$. Tissue slides were rinsed between steps with Ventana Tris-based Reaction buffer (catalog No. 950-300, Ventana Medical System). Slides were incubated at $37^{\circ} \mathrm{C}$ for 24 minutes with pAb anti - von Willebrand factor (1:2000, A0082, DAKO Corporation, Carpinteria, CA, USA), and for 32 minutes with mAb anti-podoplanin (1:50, Clone D2-40, M3619, DAKO Corporation, Carpinteria, CA, USA) followed by application of Ventana Ultraview HRP Universal Multimer (8 minutes at $37^{\circ} \mathrm{C}$ ). Diaminobenzidine was used as a chromogen and hematoxylin as a nuclear stain. Known positive tissue samples from sinonasal mucosa were also used to confirm the staining reliability of all separate staining patches. Specificity of immunohistochemistry was controlled by omitting the primary antibodies or replacing them with irrelevant antisera.

\section{Light microscopic evaluation}

Cytoplasmic staining of lymphatic and blood endothelial cells were examined with an Olympus BX41 light microscope (Olympus America Inc., Melville, NY, USA) by two observers (ST-S and TP) without knowledge of clinical status and outcome data. Cases of disagreement were discussed, and a consensus was determined for further analysis.

The vessel density was calculated in inflammatory hotspot areas and whole tissue (including hotspot and other areas) from 5 microscopic fields $\left(0.785 \mathrm{~mm}^{2} /\right.$ field). In biopsies, all microscopic fields were examined under the light microscope. Hotspot of inflammation was defined as areas of highest vascular density. These areas had also abundant inflammatory cells. The relative density of lymphatic vessels (RDLV) was calculated by dividing the mean number of lymphatic vessels per microscopic field (podoplanin) by the mean number of all vessels (vWf) per micro- scopic field. vWF is weakly expressed on lymphatic endothelium. Therefore the positive lymphatic vessel staining of vWf was assured by the lack of erythrocyte or smooth muscle within the lymphatic vessel walls. Analysis was performed at $\times 200(\times 20$ objective lens and $\times 10$ ocular lens) magnification.

\section{Data analysis}

Descriptive statistics for patients and tumour characteristics were presented in tongue cancer and in tongue hyperplasia. Fisher's exact test (2-tailed) was used to compare patient characteristics, and the correlations were analysed by Spearman rank correlation test. The comparison of the vessels densities, the relative density of lymphatic vessels in the different groups were made by Kruskal-Wallis test (more than two groups), and then by Mann Whitney U (MWU) test (two groups). Survival curves were drawn by the Kaplan-Meier method, and analysed by the logrank test. The time until revision surgery was calculated from the date of sampling to the date of the first revision endoscopic sinus surgery (ESS) and/or polypectomy, or end of March 2015 whichever came first. We sought to investigate whether vessel density has potential as a predictive marker for revision CRSsurgery. The cut-off values $\left(1,40,70,170\right.$ vessels $\left./ \mathrm{mm}^{2}\right)$ were determined by using the following criteria: detected associations by Logrank test; nearly equal-sized groups. Two-tailed P-values of $<0.05$ were considered statistically significant. Statistical analysis was carried out by the SPSS Base 15.0 Statistical Software Package (SPSS Inc., Chicago, IL, USA).

\section{Results}

\section{Patient characteristics}

The characteristics are shown in Tables 1-2. The groups from whom nasal cavity samples were taken, differed significantly in terms of age, presence of AR, AERD, asthma, previous sinus and/ or polypectomy operations(s) and medication (Table 1). The groups from whom maxillary sinus cavity samples were taken, differed significantly in terms of age, presence of AERD, asthma and medication (Table 2).

\section{Lymphatic and blood vessel density in the nasal cavity} Figure 1 presents von Willebrand factor and podoplanin expression in nasal specimens. In the whole tissue the mean density of all vessels ( $v W f$ ) was significantly lower in the polyp groups compared to control inferior turbinate (Figure $2 \mathrm{~A}$ ). In the whole tissue, the mean density of lymphatic vessels was also significantly lower in the polyp groups compared to control inferior turbinate (Figure $2 \mathrm{C}$ ).

In the inflammatory hotspot area, the mean density of all vessels (vWf) was significantly lower in the CRSwNP group, compared to control inferior turbinate (Figure $2 \mathrm{~B}$ ). In the inflammatory hotspot area, the mean density of lymphatic vessels was significantly lower in both polyp groups compared to control inferior 
Table 1. Characteristics of patients from whom nasal cavity specimens were taken.

\begin{tabular}{|c|c|c|c|c|c|c|c|c|}
\hline & & \multicolumn{2}{|c|}{ Control $n=19$} & \multicolumn{2}{|c|}{ CRSwNP $n=37$} & \multicolumn{2}{|c|}{$A C P n=9$} & \multirow[t]{2}{*}{$\mathbf{p}$} \\
\hline & & $\mathbf{n}$ & $\%$ & $\mathbf{n}$ & $\%$ & $\mathbf{n}$ & $\%$ & \\
\hline \multirow[t]{2}{*}{ Gender } & Male & 9 & 47.4 & 21 & 56.8 & 6 & 66.7 & .56 \\
\hline & Female & 10 & 52.6 & 16 & 43.2 & 3 & 33.3 & \\
\hline \multirow[t]{2}{*}{ Age } & $<40$ years & 16 & 84.2 & 8 & 21.6 & 4 & 44.4 & $<.001$ \\
\hline & $\geq 40$ years & 3 & 15.8 & 29 & 78.4 & 5 & 55.6 & \\
\hline \multirow[t]{3}{*}{$\begin{array}{l}\text { Allergic } \\
\text { rhinitis }\end{array}$} & Yes & 12 & 63.2 & 10 & 27.0 & 1 & 11.1 & .026 \\
\hline & No & 7 & 36.8 & 23 & 62.2 & 8 & 88.9 & \\
\hline & Unknown & 0 & 0.0 & 4 & 10.8 & 0 & 0.0 & \\
\hline \multirow[t]{2}{*}{ AERD } & Yes & 0 & 0.0 & 13 & 35.1 & 0 & 0.0 & .002 \\
\hline & No & 19 & 100.0 & 24 & 64.9 & 9 & 100.0 & \\
\hline \multirow[t]{3}{*}{ Asthma } & Yes & 2 & 10.5 & 19 & 51.4 & 0 & 0.0 & .001 \\
\hline & No & 17 & 89.5 & 15 & 40.5 & 9 & 100.0 & \\
\hline & Unknown & 0 & 0.0 & 3 & 8.1 & 0 & 0.0 & \\
\hline \multirow[t]{3}{*}{ Smokers } & Yes & 1 & 5.3 & 7 & 18.9 & 1 & 11.1 & .35 \\
\hline & No & 18 & 94.7 & 27 & 73.0 & 7 & 88.9 & \\
\hline & Unknown & 0 & 0.0 & 3 & 8.1 & 1 & 11.1 & \\
\hline \multirow[t]{3}{*}{ Previous op. ${ }^{1}$} & Yes & 0 & 0.0 & 10 & 70.3 & 0 & 0.0 & .013 \\
\hline & No & 19 & 100.0 & 26 & 27.0 & 9 & 100.0 & \\
\hline & Unknown & 0 & 0.0 & 1 & 2.7 & 0 & 0.0 & \\
\hline \multirow[t]{5}{*}{ Medication } & No & 19 & 100.0 & 12 & 32.4 & 6 & 66.7 & $<.001$ \\
\hline & IN CCS & 0 & 0.0 & 15 & 40.5 & 3 & 33.3 & \\
\hline & $\begin{array}{l}\text { IN CCS +PO } \\
\text { CCS }\end{array}$ & 0 & 0.0 & 5 & 13.5 & 0 & 0.0 & \\
\hline & Other $^{2}$ & 0 & 0.0 & 2 & 5.4 & 0 & 0.0 & \\
\hline & Unknown & 0 & 0.0 & 3 & 8.1 & 0 & 0.0 & \\
\hline
\end{tabular}

P-values by Fisher's exact test. Abbreviations: Control= mucosa from inferior turbinate, CRSwNP = chronic rhinosinusitis with nasal polyps, ACP $=$ antrochoanal polyp, $\mathrm{AERD}=$ Aspirin exacerbated respiratory disease, Previous op.=previous operations, ${ }^{1}$ sinus surgery and/or polypectomy, $\mathrm{CCS}=$ corticosteroid, $\mathrm{IN}=$ intranasal, $\mathrm{PO}=$ peroral, ${ }^{2}$ antihistamine.

turbinate (Figure 2D). The median vessel density and the median lymphatic vessel density both in whole tissue and in the hotspot area was not affected by the combination of intranasal and peroral treatment compared to polyp patients without corticosteroid treatment $(p=0.5, p=0.3, p=0.5, p=0.9$, respectively, by MWU test). Nor were there differences in the vessel densities between the polyp group using only intranasal corticosteroids and the polyp group without corticosteroid treatment $(p=0.1$, $p=0.8, p=0.3, p=0.5$, respectively).

The relative density of lymphatic vessels (RDLV) in the nasal

\section{cavity}

In the whole tissue, the relative density of lymphatic vessels (RDLV) was significantly lower in CRSwNP and ACP groups compared to control inferior turbinate (Figure 1E). In the inflammatory hotspot area, RDLV was also significantly lower in CRSwNP and ACP groups compared to control inferior turbinate (Figure 1F). The median RDLV both in whole tissue and in the hotspot area was not affected by the combination of intranasal and peroral treatment compared to polyp patients without corticosteroid treatment ( $p=0.3, p=0.8$, respectively). Nor were there differences in the RDLV both in the whole tissue and in 
Table 2. Characteristics of patients from whom maxillary sinus specimens were taken.

\begin{tabular}{|c|c|c|c|c|c|c|c|c|}
\hline & & \multicolumn{2}{|c|}{ Control $n=11$} & \multicolumn{2}{|c|}{ CRSsNP $n=26$} & \multicolumn{2}{|c|}{ CRSwNP $n=18$} & \multirow[t]{2}{*}{$\mathbf{p}$} \\
\hline & & $\mathbf{n}$ & $\%$ & $\mathbf{n}$ & $\%$ & $\mathbf{n}$ & $\%$ & \\
\hline \multirow[t]{2}{*}{ Gender } & Male & 4 & 36.4 & 9 & 34.6 & 7 & 38.9 & 1.00 \\
\hline & Female & 7 & 63.6 & 17 & 65.4 & 11 & 61.1 & \\
\hline \multirow[t]{2}{*}{ Age } & $<40$ years & 10 & 90.9 & 11 & 42.3 & 4 & 22.2 & .001 \\
\hline & $\geq 40$ years & 1 & 9.1 & 15 & 57.7 & 14 & 77.8 & \\
\hline \multirow[t]{2}{*}{$\begin{array}{l}\text { Allergic } \\
\text { rhinitis }\end{array}$} & Yes & 4 & 36.4 & 19 & 73.1 & 12 & 66.7 & .20 \\
\hline & No & 7 & 63.6 & 7 & 26.9 & 6 & 33.3 & \\
\hline \multirow[t]{2}{*}{ AERD } & Yes & 0 & 0.0 & 0 & 0.0 & 6 & 33.3 & .001 \\
\hline & No & 11 & 100.0 & 26 & 100.0 & 12 & 66.7 & \\
\hline \multirow[t]{2}{*}{ Asthma } & Yes & 0 & 0.0 & 9 & 34.6 & 10 & 55.6 & .004 \\
\hline & No & 11 & 100.0 & 17 & 65.4 & 8 & 44.4 & \\
\hline \multirow[t]{2}{*}{ Smokers } & Yes & 2 & 18.2 & 9 & 34.6 & 4 & 22.2 & .86 \\
\hline & No & 9 & 81.8 & 17 & 65.4 & 14 & 77.8 & \\
\hline \multirow[t]{3}{*}{ Previous op. ${ }^{1}$} & $\begin{array}{l}\text { Maxillary } \\
\text { sinus(es) }\end{array}$ & 0 & 0.0 & 3 & 11.5 & 4 & 22.2 & .085 \\
\hline & Other & 0 & 0.0 & 3 & 11.5 & 4 & 22.2 & \\
\hline & No & 11 & 100.0 & 20 & 76.9 & 10 & 55.6 & \\
\hline \multirow[t]{4}{*}{ Medication } & No & 11 & 100.0 & 13 & 50.0 & 8 & 44.4 & .006 \\
\hline & IN CCS & 0 & 0.0 & 10 & 38.5 & 5 & 27.8 & \\
\hline & $\begin{array}{l}\text { IN CCS +PO } \\
\text { CCS }\end{array}$ & 0 & 0.0 & 0 & 0.0 & 3 & 16.7 & \\
\hline & Other $^{2}$ & 0 & 0.0 & 3 & 11.5 & 2 & 11.2 & \\
\hline
\end{tabular}

Control= mucosa from inferior turbinate, CRSsNP = chronic rhinosinusitis without nasal polyps, CRSwNP = chronic rhinosinusitis with nasal polyps, $\mathrm{AERD}=$ Aspirin exacerbated respiratory disease, Previous op.=previous operations, ${ }^{1}$ sinus surgery and/or polypectomy, $C C S=$ Corticosteroid, $I N=$ intranasal, $\mathrm{PO}=$ peroral, ${ }^{2}$ antihistamine, $\mathrm{P}$-values by Fisher's exact test.

the hotspot area between the polyp group using only intranasal corticosteroids and the polyp group without corticosteroid treatment ( $p=0.9$, both).

\section{Lymphatic and blood vessel density in maxillary sinus mucosa}

Figure 1 presents von Willebrand factor and podoplanin expression in maxillary sinus specimens. In the whole tissue, the mean densities of all vessels ( $v W f$ ) and lymphatic vessels (podoplanin) did not differ between control, CRSsNP and CRSwNP groups (Figure $3 \mathrm{~A}, \mathrm{C})$. In the inflammatory hotspot area, the mean density of all vessels did not differ between the groups (Figure 2B). In the inflammatory hotspot area, the mean density of lymphatic vessels was lower in the CRSwNP group compared to the CRSsNP group (Figure 2D). The trend remained the same, when excluding patients who had undergone previous maxillary sinus surgery from the analysis $(p=0.073)$. There was also a trend that the mean density of lymphatic vessels was lower in the CRSwNP group in comparison to the control group (Figure 2D). The median vessel density both in whole tissue and in the hotspot area did not differ by the presence or absence of previous sinus surgery and/or nasal polypectomy of CRS patients $(p=1.0, p=0.3$ respectively). Accordingly the median lymphatic vessel density both in whole tissue and in the hotspot area did not differ by the presence or absence of previous sinus surgery and/or nasal polypectomy in CRS patients, $(p=0.8, p=1.0$ respectively). Neither did the use of intranasal corticosteroids by CRS patients affect the median vessel density or median lymphatic vessel density in whole tissue and in the hotspot area $(p=0.9, p=0.1, p=0.6, p=0.4$, respectively). 

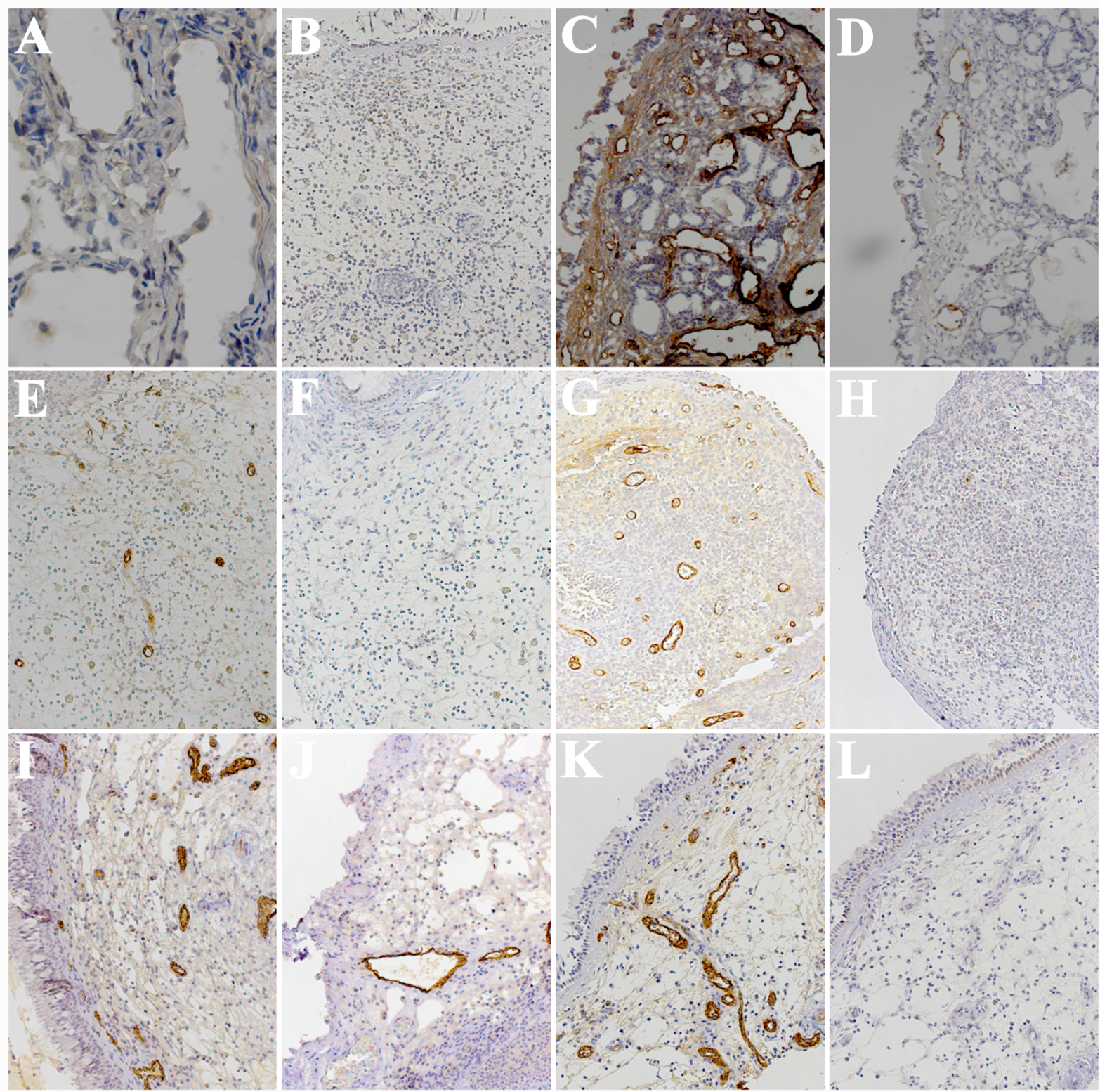

Figure 1. von Willebrand factor (vWf) and podoplanin (clone D2-40) expression in specimens from the nasal cavity (A-H) and maxillary sinus mucosa (I-L). von Willebrand factor detects all vessels and D2-40 detects lymphatic vessels only. (A) Control inferior turbinate as a negative control. (B) A polyp from a patient with chronic rhinosinusitis with nasal polyps (CRSwNP) as negative control. (C) Vessel staining with vWf in control inferior turbinate. (D) Some lymphatic vessels (stained with D2-40) in control inferior turbinate. (E) Vessel staining (vWf) in a polyp from a patient with chronic rhinosinusitis with nasal polyps and with aspirin exacerbated respiratpory disease (CRSwNP+AERD). (F) Scarce lymphatic vessels (D2-40) in a polyp from a patient with CRSwNP+AERD. (G) Vessel staining (vWf) in a inflammatory hotspot area of a polyp from a patient with CRSwNP+AERD. (H) Scarce lymphatic vessels (D2-40) in an inflammatory hotspot area of a polyp from a patient with CRSwNP+AERD. (I) Vessel staining (vWf) in control maxillary sinus mucosa. (J) Some lymphatic vessels (D2-40) in control maxillary sinus mucosa. (K) Vessel staining (vWf) in maxillary sinus mucosa of a patient with chronic rhinosinusitis without nasal polyps (CRSsNP). (L) Scarce lymphatic vessels (D2-40) in the maxillary sinus mucosa of a patient with CRSsNP. Original magnification was $\times 400(A, C) ; \times 200(B, D-L)$.

The relative density of lymphatic vessels (RDLV) in maxillary sinus mucosa

In the whole tissue, the relative density of lymphatic vessels (RDLV) did not differ between the control, CRSsNP and CRSwNP groups (Figure 3E). In the inflammatory hotspot area, RDLV was lower in the CRSwNP group compared to the CRSsNP group (Fi- gure $2 \mathrm{~F}$ ). The trend remained the same, when excluding patients who had undergone previous maxillary sinus surgery from the analysis $(p=0.078)$. There was also a trend that RDLV was lower in the CRSwNP compared to the control group (Figure 2F). Median RDLV both in whole tissue and in the hotspot area did not differ by the presence or absence of previous sinus surgery and/or 

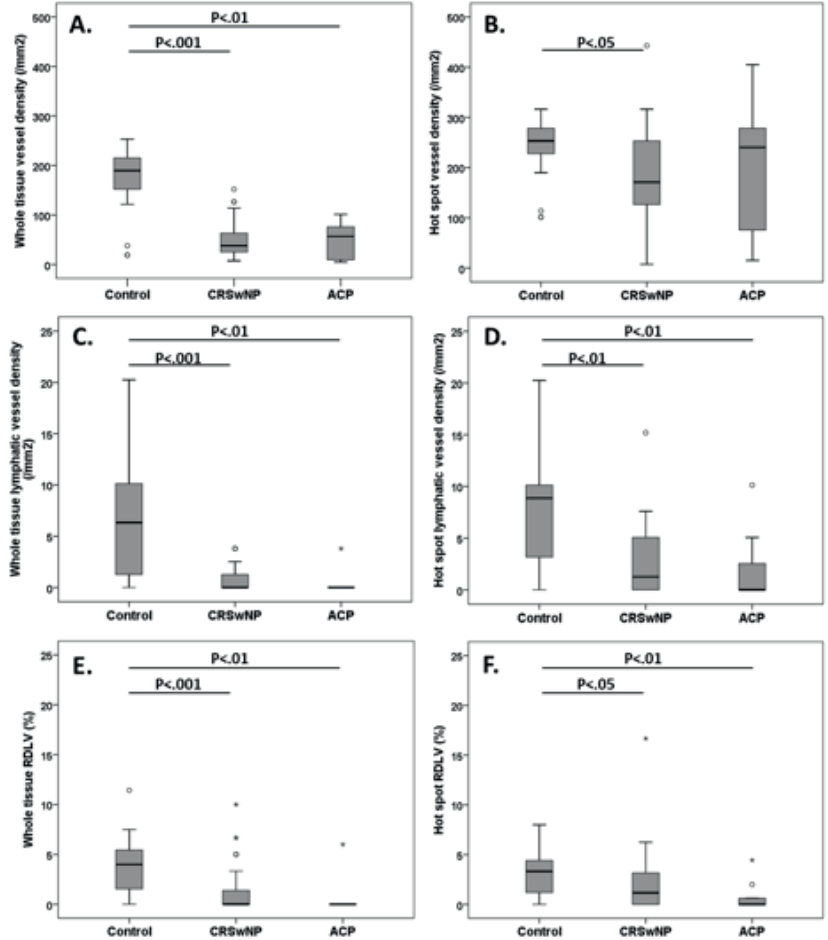

Figure 2. The density of vessels in control inferior turbinate and nasal polyp tissues. (A-B) The density of all vessels in the whole sample and in inflammatory hotspot areas detected by anti-von Willebrand factor (vWf). (C-D). The density of lymphatic vessels in the whole sample and in inflammatory hotspot areas detected by anti-podoplanin (clone D2-40). (E-F) Relative density of lymphatic vessel to all vessels (RDLV) in the whole sample and in inflammatory hotspot areas. Control= control inferior turbinate $(\mathrm{N}=19)$; $\mathrm{CRSwNP}=$ chronic rhinosinusitis with nasal polyps $(\mathrm{N}=37) ; \mathrm{ACP}=$ antrochoanal polyps $(\mathrm{N}=9)$. P-values by Mann Whitney- $\mathrm{U}$ test.

nasal polypectomy in CRS patients ( $p=0.8, p=0.9$ respectively). Neither did the use of intranasal corticosteroids in CRS patients affect median RDLV in whole tissue and in the hotspot area ( $p=0.1, p=0.3$, respectively).

Prognostic relevance of (lymphatic) vessel density We evaluated the effect of lymphatic vessel density and vessel density to the time to first revision surgery in CRS patients during the follow up of in average 9 years. High lymphatic vessel density $(\geq 1 / \mathrm{mm} 2)$ in the whole nasal polyp sample associated significantly with the need for revision endoscopic sinus surgery (ESS) and/or polypectomy, when comparing to patients with low lymphatic vessel density $(<1 / \mathrm{mm} 2)$ (Figure $4 A)$. The result remained the same when observing only the patient group with CRSwNP without ACP (Figure 4B) and the group that underwent polypectomy $\pm \mathrm{ESS}$ during sampling (Figure $4 \mathrm{C}$ ). These results remained also the same when using the values $R D L V \geq 1$ and RDLV $<1(P=.017, P=.021, P=.005$, data not shown). Nasal polyp
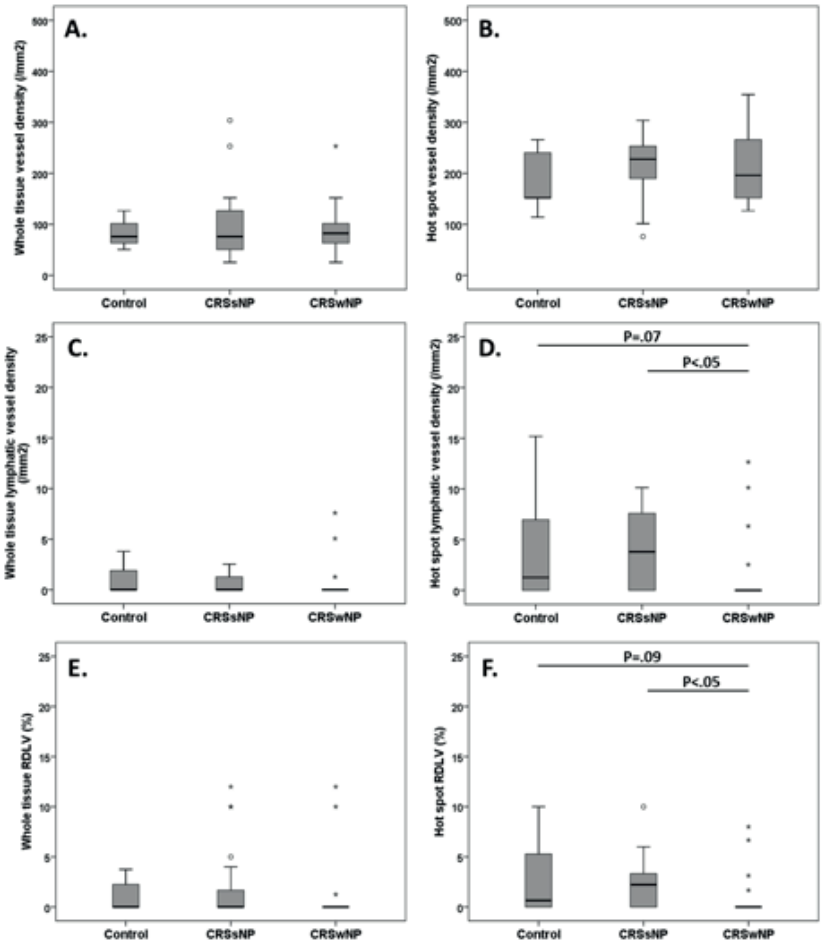

Figure 3. The density of vessels in maxillary sinus mucosa samples. (A-B) The density of all vessels in the whole sample and in inflammatory hotspot areas detected by anti-von Willebrand factor (vWf). (C-D) The density of lymphatic vessels in the whole sample and in inflammatory hotspot areas detected by anti-podoplanin (clone D2-40). (E-F) Relative density of lymphatic vessel to all vessels (RDLV) in the whole sample and in inflammatory hotspot areas. Control= control maxillary sinus mucosa $(\mathrm{N}=11)$; $C R S s N P=$ chronic rhinosinusitis without nasal polyps $(\mathrm{N}=26)$; $C R S W N P=$ chronic rhinosinusitis with nasal polyps $(N=18)$. $P$-values by Mann Whitney-U test.

vessel density did not associate significantly with the need for revision ESS and/or polypectomy. However, there was an insignificant trend that high vessel density $(\geq 40 / \mathrm{mm} 2)$ in the whole nasal polyp sample associated with the need for revision ESS and/or polypectomy, when comparing to patients with low vessel density $(<40 / \mathrm{mm} 2)$ (Figure 4D). Accordingly, high vessel density $(\geq 170 / \mathrm{mm} 2)$ in the inflammatory hotspot area of the nasal polyp sample associated insignificantly with the need for revision ESS and/or polypectomy, when comparing to patients with low vessel density $(<170 / \mathrm{mm} 2)$ (Figure $4 \mathrm{E})$. In the sample series taken from the maxillary sinus, we did not detect any significant associations between vessel or lymphatic vessel density in maxillary sinus mucosa and the need for revision ESS and/ or polypectomy. Yet, there was an insignificant trend that high vessel density ( $\geq 70 / \mathrm{mm} 2)$ in the whole maxillary sinus sample associated with the need for revision ESS and/or polypectomy, when comparing to patients with low vessel density $(<70 / \mathrm{mm} 2)$ (Figure 4E). 

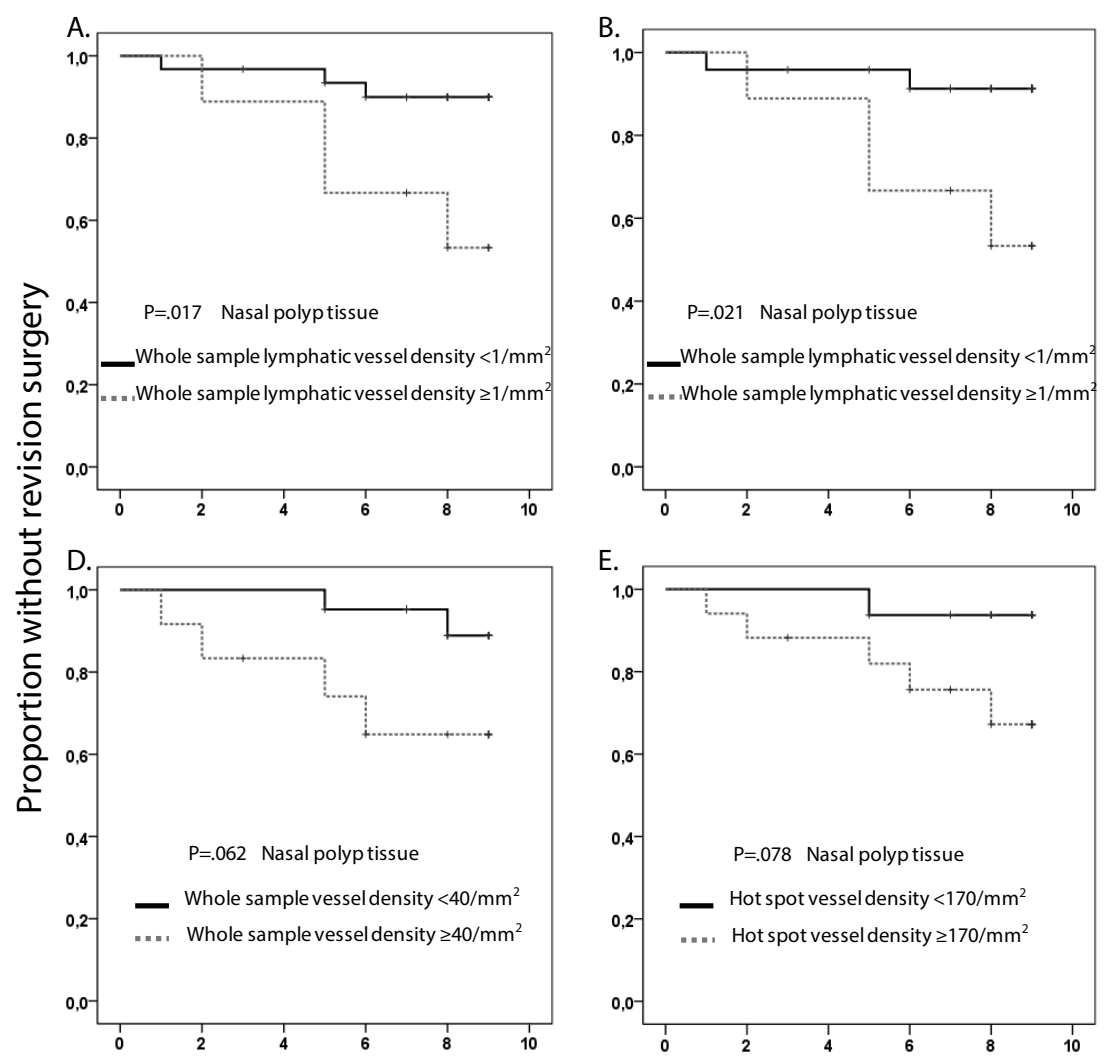

Time to revision surgery (years)
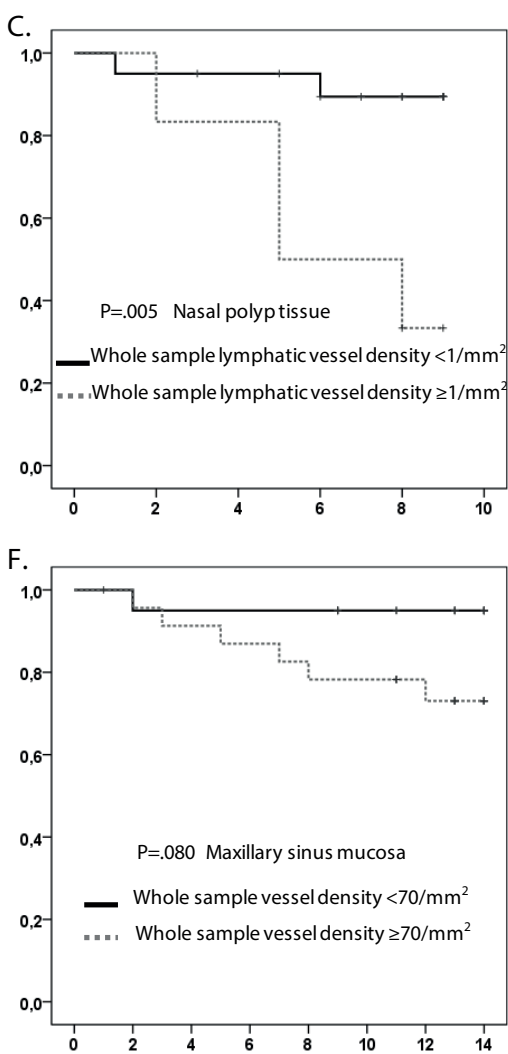

Figure 4. Prognostic relevance of the lymphatic vessel density detected by anti-podoplanin (clone D2-40); and vessel density detected by antivon Willebrand factor (vWf) to the time until the first revision endoscopic sinus surgery (ESS) and/or polypectomy in chronic rhinosinusitis (CRS) patients during follow up. A. Predictive effect of the whole sample lymphatic vessel density of nasal polyp tissue in all patients ( $N=39$ ). B. Predictive effect of the whole sample lymphatic vessel density of nasal polyp tissue only in the group with chronic rhinosinusitis with nasal polyps (CRSwNP) $(\mathrm{N}=33)$. C. Predictive effect of the whole sample lymphatic vessel density of nasal polyp tissue only in the subgroup with CRSwNP with current polypectomy \pm ESS during sampling ( $N=26$ ). D. Predictive effect of the whole sample vessel density of nasal polyp tissue in the group with CRSwNP $(\mathrm{N}=33)$. E. Predictive effect of the inflammatory hotspot vessel density of nasal polyp tissue in the group with CRSwNP ( $N=33$ ). F. Predictive effect of the whole sample vessel density of maxillary sinus mucosa in all patients $(\mathrm{N}=33)$. P-values by log rank test.

\section{Discussion}

This study was implemented to evaluate lymphatic vessel density in the nasal and maxillary sinus biopsies of patients with different types of CRS and ACP. Monoclonal anti-podoplanin, clone D2-40 is a reliable lymphatic endothelial cell marker which identifies only lymphatic endothelium ${ }^{(28,29)}$. Our most significant finding was that low lymphatic vessel density and low RDLV associate with NP and ACP tissue. This finding is in accordance with asthma studies, in which reduced lymphatic vessel distribution in patients with fatal asthma was found ${ }^{(33)}$. In view of this, impaired lymphangiogenesis has been speculated to worsen asthma outcomes ${ }^{(34,35)}$. Only few studies on lymphatic vessels in CRS are available ${ }^{(36)}$. Hosemann et al. showed that lymphatic vessels in the maxillary sinus mucosa had an anatomical orientation towards the ostium, and that they were thin but numerous in comparison to nasal lymphatic vessels. We found that the me- dian lymphatic vessel density was higher in both hotspot area and in whole tissue in maxillary sinus mucosa in comparison to nasal polyp tissue, which is in part in line with the previous observation. In contrast to us, a group found an increased number of lymphatic vessels in the nasal mucosa of patients with mild and severe persistent allergic rhinitis ${ }^{(37)}$. However, when the group studied oedematous ethmoid mucosa, they did not find any lymphatic vessels within NP tissue ${ }^{(38)}$.

In maxillary sinus mucosa, we found an insignificant trend that lymphatic vessel density and RDLV in the inflammatory hotspot area were significantly lower in CRS compared to control mucosa, whereas vessel density did not differ between CRS and control mucosa. Thus, decreased lymphatic vessels might not only be associated with the hotspot areas of polyp tissue but also with hotspot areas of the maxillary sinus mucosa in CRS 
patients. In the inflammatory hotspot area, the mean density of lymphatic vessels and RDLV were significantly lower in the CRSwNP group compared to the CRSsNP group. This would seem to point out that low lymphatic vessel density is characteristic of the CRSwNP phenotype, in other mucosal areas besides polyp tissue. Thus, decreased lymphatic vessels would probably not be solely a secondary effect to polyp formation. There might be an indirect inter-relationship between high vessel density, low lymphatic vessel density and abundant inflammatory cells in nasal polyp formation. Yet the mechanisms still remain unresolved and further studies on this are needed. In maxillary sinus mucosa, we did not detect differences between vessel densities of the whole tissue, which was in contrast to what was found in polyp tissue. This might in part be due to the fact that the size of the maxillary sinus biopsy was small and there was only little tissue that wasn't in the inflammatory hotspot area.

A study group did not find differences in number, size and length density of lymphatic vessels in normal and oedematous ethmoid sinus mucosa ${ }^{(38)}$. Western blot also showed no differences in their expression levels. The authors concluded that lymphangiogenesis does not occur in oedematous ethmoid sinus mucosa, which may not reuptake interstitial fluid efficiently in inflammatory conditions, resulting in the formation of mucosal oedema in chronic inflammation ${ }^{(38)}$.

We detected differences in vessel densities and RDLV, when observing whole tissue and inflammatory hotspot area. Histomorphological findings in NPs include tissue with few cells and vessels; blood vessels found in NPs have been described as being "immature" and lacking normal innervation ${ }^{(39)}$. In regards to this, it would seem to be important to study hotspot areas i.e. NP and whole tissue areas i.e. inferior or middle turbinate, separately.

In animal models, lymphangiogenesis has been shown to associate with chronic airway diseases. In a murine asthma model, lymphangiogenesis was found to be increased in obese mice and during allergen challenge in sensitized mice in visceral and subcutaneous adipose tissue ${ }^{(40)}$. Similarly, in a murine mycoplasma pulmonis driven model of chronic airway inflammation, lymphatic sprouting was completely inhibited by a long course of antibiotics, but lymphatic vessels regressed only partly in comparison to the almost complete regression of blood vessels (34). In humans, lymphangiogenesis has been documented to increase in idiopathic pulmonary fibrosis ${ }^{(41)}$.

We found that vessel density was lower in nasal polyp and ACP tissue. This is supported by the study in which a reduced number of $\mathrm{CD} 34+$ vessels was observed in various NP subtypes, including cystic fibrosis patients and ACP polyps, and especially in patients with $A E R D{ }^{(42,43)}$. VEGF is one of the most important molecules controlling inflammation-driven angiogenesis. An association was found between pro-angiogenic factor CD105 (endoglin) staining intensity and an eosinophilic histological pattern in CRSwNP adult patients ${ }^{(44)}$. Increased angiogenesis and density of blood vessels as documented by positive CD34 and pro-angiogenic factor VEGF has been reported respectively in adult CRSwNP and ACP patients ${ }^{(17)}$. This is in contrast with our findings, which could in part be due to different control tissues. We used inferior turbinate from subjects with/without allergic rhinitis. Hirshoren et al. used middle turbinate tissue from nonatopic patients that underwent skull base surgery due to cerebrospinal fluid leak or pituitary gland operation ${ }^{(17)}$. In children, higher VEGF and vessel density has been demonstrated in the hotspot areas of polyp tissue compared to that of the ethmoid sinus mucosa of CRSsNP patients contrasts with our findings, which could be explained by the differences in studied populations and by the fact that Hu et al. ${ }^{(18)}$ used ethmoid sinus mucosa and we used maxillary sinus mucosa of CRSsNP patients.

We demonstrated, that NP vessel density did not associate significantly with need for revision ESS and/or polypectomy. Ottaviano et al also found that neo-angiogenesis, as documented by CD105 staining, was not predictive of NP recurrence ${ }^{(39)}$. Interestingly, we found that an increased amount of lymphatic vessel density in the whole NP sample associated significantly with an increased need for revision ESS and/or polypectomy. Thus, although reduced lymphatic vessel density associates with CRSwNP it seems to, however, predict better long-term control of CRSwNP. Thus, it could be possible that lymphatic vessel density in nasal polyp tissue is not a primary cause of recurrent polyp growth. Moreover, it could be speculated, that induced lymphangiogenesis could have therapeutic potential in inhibiting primary growth of CRSwNP, but after polypectomy, stimulated lymphangiogenesis might not have therapeutic potential in reducing the need for revision surgery. Yet, this would require further studies to be proven.

We acknowledge the number of patients was small and control nasal cavity samples were from inferior turbinate instead of middle turbinate. In addition, no corresponding samples from the same patient's polyp tissue, middle turbinate and sinus cavity were available in this study. The small number of samples did not allow us to evaluate the effect of confounding factors, such as AERD, asthma and intranasal or peroral corticosteroid treatment. Vessel densities in maxillary sinus mucosa might be affected by previous sinus surgery, yet the small sample size limited our possibilities to completely evaluate this. On the other hand, we were able to detect the associations despite the ingroup heterogeneity. Despite the cohort setup, the study design did not fully make it possible to demonstrate causal association between low lymphatic vessel densities to polyp formation. 
The low number of subjects limited the possibilities to evaluate whether the vessel or lymphatic vessel densities and the used cut-off values would be clinically feasible predictive markers for recurrent CRS-surgery.

\section{Conclusion}

The study suggests that low absolute and relative lymphatic vessel density associate to nasal polyps and ACP, and to a reduced need for revision surgery. Further studies with greater population size would be needed to prove these findings and to observe, which factors lead to reduced lymphatic vessel density in CRSwNP and whether lymphatic vessel density is a cause or a secondary event for polyp growth.

\section{Acknowledgements}

The study was supported by the Competitive Research Funding of the Tampere Medical Research Fund of Tampere University Hospital, and in part by research grants from the Finnish Medical
Foundation, Finnish Association of Otorhinolaryngology and Head and Neck Surgery, Jane and Aatos Erkko Foundation, Paulo Foundation, Väinö and Laina Kivi Foundation, Minerva Foundation, Yrjö Jahnsson Foundation and Dentists of Helsinki Region. We thank Eini Eskola, Marja-Leena Oksanen, Marja-Leena Koskinen, Raija Hukkila, Teemu Honkanen and Mikko Lehtonen for excellent assistance.

\section{Authorship contribution}

A.L., S.T.-S., T.P. and M.S. planned the study, performed laboratory tests and analyses, and wrote the manuscript. J.M., M.R. and T.T. provided samples. J.R., R.R., J.H., H.H. and A.R. provided expertise in analyses. All authors critically reviewed the manuscript.

\section{Conflict of interest}

No conflict of interest.

\section{References}

1. Fokkens WJ, Lund VJ, Mullol J, Bachert C, Alobid I, Baroody F, et al. European Position Paper on Rhinosinusitis and Nasal Polyps 2012. Rhinol Suppl 2012 Mar;(23)(23):3 p preceding table of contents, 1-298.

2. Kim YS, Kim NH, Seong SY, Kim KR, Lee GB, Kim KS. Prevalence and risk factors of chronic rhinosinusitis in Korea. Am J Rhinol Allergy 2011 May-Jun;25(3):117-121.

3. Pilan RR, Pinna FR, Bezerra TF, Mori RL, Padua FG, Bento RF, et al. Prevalence of chronic rhinosinusitis in Sao Paulo. Rhinology 2012 Jun;50(2):129-138.

4. Jarvis D, Newson R, Lotvall J, Hastan D Tomassen $\mathrm{P}$, Keil T, et al. Asthma in adults and its association with chronic rhinosinusitis: the GA2LEN survey in Europe. Allergy 2012 Jan;67(1):91-98

5. Jankowski R, Bouchoua F, Coffinet L, Vignaud JM. Clinical factors influencing the eosinophil infiltration of nasal polyps. Rhinology 2002 Dec;40(4):173-178.

6. Kakoi H, Hiraide F. A histological study of formation and growth of nasal polyps. Acta Otolaryngol 1987 Jan-Feb;103(1-2):137-144.

7. Ishitoya J, Sakuma Y, Tsukuda M Eosinophilic chronic rhinosinusitis in Japan. Allergol Int 2010 Sep;59(3):239-245.

8. Stevens WW, Ocampo CJ, Berdnikovs S, Sakashita M, Mahdavinia M, Suh L, et al. Cytokines in Chronic Rhinosinusitis. Role in Eosinophilia and Aspirin-exacerbated Respiratory Disease. Am J Respir Crit Care Med 2015 Sep 15;192(6):682-694.

9. Zhang N, Van Zele T, Perez-Novo C, Van Bruaene N, Holtappels G, DeRuyck N, et al. Different types of T-effector cells orchestrate mucosal inflammation in chronic sinus disease. J Allergy Clin Immunol 2008 Nov;122(5):961-968.
10. Cao PP, Li HB, Wang BF, Wang SB, You XJ, Cui $\mathrm{YH}$, et al. Distinct immunopathologic characteristics of various types of chronic rhinosinusitis in adult Chinese. J Allergy Clin Immunol 2009 Sep;124(3):478-84, 484.e1-2.

11. Meng J, Zhou P, Liu Y, Liu F, Yi X, Liu S, et al. The development of nasal polyp disease involves early nasal mucosal inflammation and remodelling. PLoS One 2013 Dec 10;8(12):e82373.

12. Baba S, Kagoya R, Kondo K, Suzukawa M Ohta K, Yamasoba T. T-cell phenotypes in chronic rhinosinusitis with nasal polyps in Japanese patients. Allergy Asthma Clin Immunol 2015 Nov 19;11:33-015-0100-2. eCollection 2015.

13. Yaman H, Yilmaz S, Karali E, Guclu E, Ozturk O. Evaluation and management of antrochoanal polyps. Clin Exp Otorhinolaryngol 2010 Jun;3(2):110-114.

14. Knor M, Tziridis K, Agaimy A, Zenk J, Wendler O. Human Papillomavirus (HPV) Prevalence in Nasal and Antrochoanal Polyps and Association with Clinical Data. PLoS One 2015 Oct 28;10(10):e0141722.

15. Tatti O, Gucciardo E, Pekkonen P Holopainen $T$, Louhimo R, Repo P, et al. MMP16 Mediates a Proteolytic Switch to Promote Cell-Cell Adhesion, Collagen Alignment, and Lymphatic Invasion in Melanoma. Cancer Res 2015 May 15;75(10):2083-2094.

16. Audet N, Beasley NJ, MacMillan C, Jackson DG, Gullane PJ, Kamel-Reid S. Lymphatic vessel density, nodal metastases, and prognosis in patients with head and neck cancer. Arch Otolaryngol Head Neck Surg 2005 Dec;131(12):1065-1070

17. Hirshoren N, Neuman T, Gross M, Eliashar R. Angiogenesis in chronic rhinosinusitis with nasal polyps and in antrochoanal polyps.
Inflamm Res 2011 Apr;60(4):321-327.

18. Hu KH, Lee FP, Cheng YJ, Huang HM Vascular endothelial growth factor and children featuring nasal polyps. Int J Pediatr Otorhinolaryngol 2007 Jan;71(1):23-28.

19. Harkness LM, Ashton AW, Burgess JK. Asthma is not only an airway disease, but also a vascular disease. Pharmacol Ther 2015 Apr;148:17-33.

20. Keglowich LF, Borger P. The Three A's in Asthma - Airway Smooth Muscle, Airway Remodeling \& Angiogenesis. Open Respir Med J 2015 Jun 17;9:70-80.

21. Denis CV. Molecular and cellular biology of von Willebrand factor. Int J Hematol 2002 Jan;75(1):3-8

22. Yang $X$, Sun HJ, Li ZR, Zhang $H$, Yang WJ, Ni $B$, et al. Gastric cancer-associated enhancement of von Willebrand factor is regulated by vascular endothelial growth factor and related to disease severity. BMC Cancer 2015 Feb 21;15:80-015-1083-6.

23. Wada H, Shiozawa M, Sugano N, Morinaga S, Rino Y, Masuda M, et al. Lymphatic invasion identified with D2-40 immunostaining as a risk factor of nodal metastasis in T1 colorectal cancer. Int J Clin Oncol 2013 Dec;18(6):1025-1031.

24. Kahn HJ, Bailey D, Marks A. Monoclonal antibody D2-40, a new marker of lymphatic endothelium, reacts with Kaposi's sarcoma and a subset of angiosarcomas. Mod Pathol 2002 Apr;15(4):434-440.

25. Fogt F, Zimmerman RL, Ross $H M$, Daly $T$, Gausas RE. Identification of lymphatic vessels in malignant, adenomatous and normal colonic mucosa using the novel immunostain D2-40. Oncol Rep 2004 Jan;1 1(1):4750.

26. Miettinen $M$, Lindenmayer $A E$, Chaubal A. Endothelial cell markers CD31, CD34, 
and $\mathrm{BNH} 9$ antibody to $\mathrm{H}$ - and $\mathrm{Y}$-antigens-evaluation of their specificity and sensitivity in the diagnosis of vascular tumors and comparison with von Willebrand factor. Mod Pathol 1994 Jan;7(1):82-90.

27. Erhard H, Rietveld FJ, Brocker EB, de Waal RM, Ruiter DJ. Phenotype of normal cutaneous microvasculature. Immunoelectron microscopic observations with emphasis on the differences between blood vessels and lymphatics. J Invest Dermatol 1996 Jan;106(1):135-140.

28. Michikawa C, Uzawa N, Kayamori K, Sonoda I, Ohyama Y, Okada N, et al. Clinical significance of lymphatic and blood vessel invasion in oral tongue squamous cell carcinomas. Oral Oncol 2012 Apr;48(4):320-324.

29. Marks A, Sutherland DR, Bailey D, Iglesias J, Law J, Lei M, et al. Characterization and distribution of an oncofetal antigen (M2A antigen) expressed on testicular germ cell tumours. Br J Cancer 1999 May;80(3-4):569578.

30. Agarwal D, Pardhe N, Bajpai M, Gupta S, Mathur N, Vanaki SS, et al. Characterization, Localization and Patterning of Lymphatics and Blood Vessels in Oral Squamous Cell Carcinoma: A Comparative Study Using D2-40 and CD-34 IHC Marker. J Clin Diagn Res 2014 Oct;8(10):ZC86-9.

31. Honkanen $T$, Luukkainen $A$, Lehtonen $M$, Paavonen T, Karjalainen J, Hurme M, et al. Indoleamine 2,3-dioxygenase expression is associated with chronic rhinosinusitis with nasal polyps and antrochoanal polyps. Rhinology 2011 Aug;49(3):356-363.

32. Toppila-Salmi SK, Myller JP, Torkkeli TV Muhonen JV, Renkonen JA, Rautiainen ME, et al. Endothelial L-selectin ligands in sinus mucosa during chronic maxillary rhinosi- nusitis. Am J Respir Crit Care Med 2005 Jun 15;171(12):1350-1357

33. Ebina M. Remodeling of airway walls in fatal asthmatics decreases lymphatic distribution; beyond thickening of airway smooth muscle layers. Allergol Int 2008 Jun;57(2):165-174.

34. Baluk P, Tammela T, Ator E, Lyubynska N, Achen MG, Hicklin DJ, et al. Pathogenesis of persistent lymphatic vessel hyperplasia in chronic airway inflammation. J Clin Invest 2005 Feb;1 15(2):247-257.

35. Detoraki A, Granata F, Staibano S, Rossi FW Marone G, Genovese A. Angiogenesis and lymphangiogenesis in bronchial asthma. Allergy 2010 Aug;65(8):946-958.

36. Hosemann W, Kuhnel T, Burchard AK Werner JA. Histochemical detection of lymphatic drainage pathways in the middle nasal meatus. Rhinology 1998 Jun;36(2):5054.

37. Kim TH, Lee JY, Lee HM, Lee SH, Cho WS, Ju $\mathrm{YH}$, et al. Remodelling of nasal mucosa in mild and severe persistent allergic rhinitis with special reference to the distribution of collagen, proteoglycans, and lymphatic vessels. Clin Exp Allergy 2010 Dec;40(12):17421754

38. Kim $\mathrm{TH}$, Lee $\mathrm{SH}$, Lee $\mathrm{HM}$, Lee $\mathrm{SH}$, Jung $\mathrm{HH}$, Cho WS, et al. D2-40 immunohistochemical assessment of lymphangiogenesis in normal and edematous sinus mucosa and nasal polyp. Laryngoscope 2007 Mar;117(3):442-446.

39. Ottaviano G, Cappellesso R, Mylonakis Lionello M, Favaretto N, Giacomelli L, et al. Endoglin (CD105) expression in sinonasa polyposis. Eur Arch Otorhinolaryngol 2015 Nov;272(11):3367-3373

40. Ramalho R, Almeida J, Fernandes R, Costa
R, Pirraco A, Guardao L, et al. Neurokinin-1 receptor, a new modulator of lymphangiogenesis in obese-asthma phenotype. Life Sci 2013 Aug 6:93(4):169-177.

41. Yamashita M, Mouri T, Niisato M, Nitanal $H$, Kobayashi $H$, Ogasawara M, et al. Lymphangiogenic factors are associated with the severity of hypersensitivity pneumonitis. BMJ Open Respir Res 2015 Sep 29;2(1):e000085-2015-000085. eCollection 2015.

42. Ebbens FA, Toppila-Salmi SK, Renkonen JA, Renkonen RL, Mullol J, van Drunen CM, et al. Endothelial L-selectin ligand expression in nasal polyps. Allergy 2010 Jan;65(1):95102.

43. Cauna N, Manzetti GW, Hinderer KH, Swanson EW. Fine structure of nasal polyps. Ann Otol Rhinol Laryngol 1972 Feb;81(1):41-58.

44. Fonsatti $E$, Altomonte M, Nicotra MR, Natali PG, Maio M. Endoglin (CD105): a powerful therapeutic target on tumor-associated angiogenetic blood vessels. Oncogene 2003 Sep 29;22(42):6557-6563.

\section{Sanna Toppila-Salmi \\ Haartman Institute \\ University of Helsinki \\ PO Box 21 \\ Haartmaninkatu 3 \\ Helsinki Fl 00014 \\ Finland}

E-mail: sanna.salmi@helsinki.fi 